\title{
BMJ Open Protocol for Care After Lymphoma (CALy) trial: a phase II pilot randomised controlled trial of a lymphoma nurse-led model of survivorship care
}

\author{
Karen Taylor, ${ }^{1,2}$ David Joske, ${ }^{3,4}$ Max Bulsara, ${ }^{5}$ Caroline Bulsara, ${ }^{2}$ \\ Leanne Monterosso ${ }^{2,6,7}$
}

To cite: Taylor K, Joske D, Bulsara M, et al. Protocol for Care After Lymphoma (CALy) trial: a phase II pilot randomised controlled trial of a lymphoma nurse-led model of survivorship care. BMJ Open 2016;6:e010817. doi:10.1136/bmjopen-2015010817

- Prepublication history for this paper is available online. To view these files please visit the journal online (http://dx.doi.org/10.1136/ bmjopen-2015-010817).

Received 16 December 2015 Revised 23 March 2016 Accepted 28 April 2016

CrossMark

For numbered affiliations see end of article.

\section{Correspondence to}

Karen Taylor;

Karen.Taylor@health.wa.gov. $\mathrm{au}$

\section{ABSTRACT}

Introduction: Lymphoma is the sixth most common cancer diagnosed in Australia and internationally. Owing to the aggressive nature of the disease and intensity of treatment, survivors face long-term effects that impact on quality of life. Current models of followup post-treatment fail to address these complex issues. Given that $74 \%$ of patients with lymphoma cancer now survive 5 years beyond diagnosis and treatment, it is important to address this gap in care.

Aim: To determine self-reported informational and practical needs, anxiety, depression, stress, coping and empowerment at baseline, 3 and 6 months.

Methods and analysis: A pilot randomised controlled trial will test the effect of a nurse-led lymphoma survivorship clinic compared with usual post-treatment care at a large tertiary cancer centre in Western Australia. The intervention will comprise three face-to-face appointments with delivery of tailored resources, a survivorship care plan and treatment summary (SCP TS). The SCP TS will be given to the participant and general practitioner (GP). Intervention participants will be interviewed at completion to explore the perceived value of the intervention components and preferred dose. An evaluation developed for GPs will assess receipt and use of SCP TS. The primary intent of analysis will be to address the feasibility of a larger trial and requisite effect and sample size.

Ethics and dissemination: Ethics approval has been granted by the University of Notre Dame Australia and Sir Charles Gairdner Hospital in Western Australia.

Peer-reviewed publications and conference

presentations will report the results of this phase II trial.

Trial registration number: ANZCTRN126150005 30527; Pre-results.

\section{INTRODUCTION}

Lymphoma is a general term for over 20 blood cancers that originate from $\mathrm{T}$ and $\mathrm{B}$ cells in the lymphatic system ${ }^{1}$ where

\section{Strengths and limitations of this study}

- This is the first randomised controlled trial of a nurse-led model of survivorship care for patients completing treatment for lymphoma cancer in Western Australia.

- This trial will test a developed lymphoma-specific survivorship care plan and treatment summary.

- As a pilot study, it is designed to provide preliminary data on the efficacy and feasibility of a nurse-led survivorship intervention for the purposes of planning a phase III study.

lymphocytes undergo a malignant change and multiple uncontrollably. Lymphomas, when combined, represent the sixth most commonly diagnosed cancer worldwide, ${ }^{2}$ with Hodgkin's lymphoma (HL) and non-Hodgkin's lymphoma (NHL) the two main forms. HL represents $11.5 \%$ of all lymphomas and is the third most common cancer in the adolescent and young adult population. ${ }^{1}$ With the exception of HL, incidence increases with age; thus, NHL is predominantly a cancer of the older population (over 65 years). ${ }^{13}$

The incidence of lymphoma in Australia is increasing, with a projected diagnosis of 5680 cases in 2015 . This will equate to $4.5 \%$ of all cancer cases. ${ }^{4}$ In Australia, the overall survival rate has improved, and $\sim 74 \%$ of people diagnosed with lymphoma are reported as being alive at 5 years compared with $49 \%$ in the $1980 \mathrm{~s}^{4}$ Despite these encouraging results, ${ }^{5}$ this group of cancers remain understudied and subsequently under-represented in survivorship care. ${ }^{6}$

Lymphoma treatment regimens commonly involve aggressive high-dose chemotherapy and/or targeted therapy agents, radiotherapy 
and haematopoietic stem cell transplants. ${ }^{7}$ Such treatments result in distressing long-term and late physical, practical and psychosocial effects, which can produce ongoing unmet needs. These needs relate to physical and psychosocial impacts such as fear of recurrence, fatigue, poor nutrition, exercise, fertility, relationship, financial, employment and insurance issues. Furthermore, these patients commonly experience related health problems earlier than the general population $^{9}$ and are at risk of specific late effects. Cardiovascular disease is particularly pertinent in this cohort due to chemotherapy combinations and cumulative dosing ${ }^{10}{ }^{11}$ as well as mediastinal radiotherapy. ${ }^{12} 13$ Patient health and lifestyle behaviours, for example, smoking, likewise have an effect on disease development. ${ }^{11}$ Patients with lymphoma have an increased relative risk of second cancers, higher when diagnosed at a younger age $\mathrm{e}^{14} 15$ and further elevated when treatment includes radiotherapy. ${ }^{11}{ }^{12}$ The potential for the development of bone marrow disease is greater in the first decade; however, unlike second cancer risk, this decreases and then plateaus in the second decade. ${ }^{11}$ Patients who require a haematopoietic stem cell transplant have additional transplant-related late-effect risks. ${ }^{16}{ }^{17}$ Although patients may be unable to modify some late-effect risks, awareness of relevant potential late effects may ensure timely follow-up for symptomology. ${ }^{11}$

The traditional model of haematological cancer care follow-up has largely been haematologist led within the acute hospital setting. ${ }^{8}$ Information at treatment completion is often inadequate, ${ }^{18}{ }^{19}$ with a lack of clear guidelines for the ongoing management of survivors. ${ }^{20}$ This has led to an emerging focus on redesigning survivorship follow-up care and delivery.

Lobb et $a l^{21}$ demonstrated patient-reported needs among Western Australian haematological cancer survivors $(\mathrm{n}=66)$ not addressed during routine follow-up posttreatment completion and thereby classified as unmet needs. Almost two-thirds of respondents (59\%) would have found it helpful to talk with a health professional at treatment completion. A recent qualitative study conducted by the authors with lymphoma and leukaemia cancer survivors $(n=19)$ in Western Australia ${ }^{22}$ found unmet needs relating to information, practical support, coping strategies and transitioning from active treatment into the survivorship phase. Findings suggested that tailored, end-of-treatment interventions should form a key component of survivorship care. Participants suggested a cancer coordinator nurse as an important element to initiate and transition patients into the survivorship phase.

Nurse-led models of care have demonstrated potentially satisfactory outcomes ${ }^{23-25}$ and are proposed as an acceptable pathway to transition into the survivorship phase. ${ }^{26}$ A dedicated nurse-led survivorship clinic to administer patient-centred survivor-specific needs assessments is an important aspect of survivorship care to address patient concerns and empower survivors to manage their own health and ongoing symptoms. ${ }^{27-30}$
Empowering patients enables them to become more responsible for the management of their own health and well-being, and can contribute to the influence and control patients have over their own health which has the advantage of improving quality of life. ${ }^{31}{ }^{32}$ Bandura's theory of self-efficacy, ${ }^{33}$ the principal concept in selfmanagement education, teaches patients to identify their problems and provides skills in decision-making and developing an appropriate action plan. ${ }^{31}$ It is anticipated that increasing empowerment and providing healthy lifestyle resources will result in a reduction in the patient-perceived need for support from the healthcare system. $^{31}$

Survivorship care plans (SCPs) and treatment summaries (TS) have been recommended as facilitators to deliver holistic survivorship follow-up care by the Institute of Medicine ${ }^{34}$ the American Society of Clinical Oncology, ${ }^{35}$ the UK National Cancer Survivorship Initiative $^{36}$ and the proposed Clinical Oncology Society of Australia survivorship guidelines. ${ }^{37} \mathrm{~A}$ personalised SCP would guide follow-up care by including recommendations, information and resources for surveillance, screening of potential long-term and late effects and health-promoting behaviours. ${ }^{38}$ The TS would comprehensively summarise information on diagnosis and treatments. ${ }^{39}{ }^{40}$ Cancer nurses have established expertise in the areas of health promotion, information, support and resource provision, ${ }^{41}$ and therefore can develop and disseminate SCPs and TS to facilitate communication between the survivor, specialist and primary care.

\section{AIM}

The aim of the Care After Lymphoma (CALy) study is to develop and empirically test an evidence-based nurse-led lymphoma survivorship clinic to transition participants into the survivorship phase, using a pilot randomised controlled trial (RCT) design. This phase II trial of an intervention is aimed at reducing the immediate and long-term physical and psychosocial consequences of haematological cancer treatment and to enable the participant to return to normal functioning sooner. The nurse-led lymphoma survivorship clinic has three core components: (1) needs assessments to determine individual informational or practical issues or concerns; (2) provision of a tailored survivorship care plan and treatment summary to enhance communication between the participant and all other health professionals with whom the patient has contact post-treatment; and (3) provision of individualised evidence-based education, information and resources to address patient-reported needs, likely post-treatment physical and emotional concerns and maximising participant involvement in healthy lifestyle behaviours. The aims are aligned with the Australian national research priority for preventative healthcare to reduce comorbid diseases in cancer survivors.

The Medical Research Council framework for the development and evaluation of complex interventions 
has guided the development of this trial. ${ }^{42}{ }^{43}$ The evaluation of a model for nurse-led evidence-based survivorship care will provide level II baseline data to endorse the suitability of outcome measures, establish acceptability of the intervention and randomisation, provide recruitment and attrition rates, support hypothesis development and calculate sample sizes for future phase III multisite RCTs. In addition, it will add psychometric information on the Short-Form Survivor Unmet Needs Survey (SF-SUNS) and will provide data on a test-retest analysis.

\section{RESEARCH QUESTIONS}

The following research questions guide this pilot RCT:

1. Do participants assigned to the nurse-led lymphoma survivorship clinic demonstrate a reduction in perceived unmet informational and practical needs compared with those randomly assigned to usual care?

2. Do participants assigned to the nurse-led lymphoma survivorship clinic demonstrate a reduction in selfreported anxiety, depression and stress and an increase in patient self-management behaviours compared with participants randomly assigned to usual care?

3. What is the perceived efficacy and value of the nurse-led lymphoma survivorship clinic from the perspective of a subset of survivors in the intervention group?

4. To what extent does the provision of an SCP TS to general practitioners (GPs) improve the communication between the treating hospital, GP and the participant?

5. Does the SF-SUNS demonstrate stability and reliability over time?

\section{METHODS}

\section{Design}

The evidence to support the development of the phase II CALy trial comprised a qualitative study using a focus group methodology with lymphoma, leukaemia and multiple myeloma survivors. ${ }^{22}$ The evidence also encompassed three systematic reviews regarding models of haematological survivorship care; SCPs and TS in patients with haematological cancer; and tools used to assess the informational and practical needs of acute leukaemia and lymphoma survivors. ${ }^{8} 3844$ Information gained from this preliminary work guided the development of intervention components and the operationalisation of the feasibility and acceptability of a nurse-led RCT.

The RCT framework has been developed using the Consolidated Standards of Reporting Trials (CONSORT) statement and checklist. ${ }^{45} 46$ Outcomes will be measured using validated needs assessment instruments. Reporting will include inclusion and exclusion criteria; missing data; dropout; and early closure of the trial if required (figure 1). The survivorship cancer nurse coordinator $(\mathrm{CNC})$ is a specialist cancer nurse with an extensive haematology nursing background and formal counselling qualifications, including motivational interviewing techniques.

\section{Population and setting}

A convenience sample of patients with lymphoma cancer from a specialised haematology department in a comprehensive cancer centre of a large acute tertiary hospital in Perth, Western Australia, will be used. Follow-up by a haematologist occurs every 3 months for the first 12 months. The nurse-led survivorship clinic intervention will be an additional care activity to the medical haematology follow-up and will involve three appointments over 6 months. It will start at 3 months post-treatment completion and cease at 9 months post-treatment.

\section{Inclusion criteria}

1. Pathologically confirmed new diagnosis of HL or NHL.

2. Completed first-line curative intent chemotherapy or second-line curative intent autologous stem cell transplant within the previous 3 months.

3. No evidence of lymphoma disease on mid-treatment interim positron emission tomography (PET) scan or post-treatment PET scan where these are performed.

4. Able to understand and read English.

5. Over 18 years.

\section{Exclusion criteria}

1. Diagnosis of other haematological malignancy.

2. Did not undergo chemotherapy.

3. Further treatment and follow-up at another hospital.

4. Intellectually impaired or experiencing an acute mental health condition that precludes the ability to provide informed consent.

5. Comorbid condition requiring monthly visits with GP. To measure selection bias, minimal data will be completed on eligible participants who decline to participate. Reasons for refusal will be recorded to gain valuable information for future research.

\section{Recruitment}

Identification of eligible participants will be undertaken by haematology clinicians who will provide details to the survivorship CNC. Ongoing education of clinicians (haematologists and nurses) regarding all aspects of the study, its progress and recruitment will facilitate cooperation and support. Eligible participants will be met after treatment completion by the $\mathrm{CNC}$ who will discuss the study and provide a Participant Information and Consent Form (PICF). Consenting participants randomised to the intervention group $(n=30)$ will be offered the opportunity to consent to a qualitative interview at completion of all time points. Approximately one-third of participants $(n=10)$ will be required for this phase. Participants' names and contact details will be entered 
Figure 1 Trial flow chart. DASS-21, Depression Anxiety

Stress Scale; GP, general practitioner; Mini-MACM,

Mini-Mental Adjustment to Cancer Scale; PES, Patient

Empowerment Scale; PET, positron emission tomography; SCP TS, survivorship care plan and treatment summary; SF-SUNS, Short-Form Survivor Unmet Needs Survey.

\begin{tabular}{|l|}
\hline Identification of \\
eligible patients \\
Inclusion: understand \\
\hline English; completed \\
curative intent \\
chemotherapy or \\
autologous transplant for \\
new Hodgkin or non- \\
Hodgkin lymphoma; no \\
evidence of lymphoma \\
on PET scan; over 18 \\
years of age. \\
Exclusion: diagnosis of \\
other haematological \\
malignancy; undergoing \\
active treatment; \\
intellectually impaired; \\
experiencing an acute \\
mental health condition \\
that precludes ability to \\
provide informed \\
consent; comorbid \\
condition requiring \\
monthlv visits with GP \\
\hline
\end{tabular}

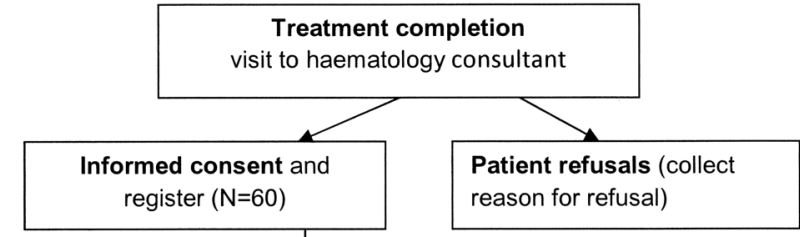

Intervention group $(\mathrm{N}=30)$
Nurse-led lymphoma survivorship clinic

- Consultation with Cancer Nurse Coordinator to normalise end of treatment concerns

- Delivery and discussion of tailored SCP TS

- Discussion of identified needs and goal setting

- Tailored resource pack

- SCP TS sent to GP

\begin{tabular}{|c|c|c|}
\hline \multirow{2}{*}{\multicolumn{2}{|c|}{$\begin{array}{l}\text { Time } 2 \text { ( } 3 \text { months after baseline) } \\
\text { - } \quad \text { Consultation in nurse-led clinic } \\
\text { - } \quad \text { Discuss previously identified needs } \\
\text { - } \quad \text { Apply measures: SF-SUNS; DASS21; MiniMac; PES } \\
\text { - } \quad \text { Encourage self-empowerment strategies } \\
\text { - } \quad \text { Encourage follow-up with GP }\end{array}$}} & \multirow[t]{2}{*}{$\begin{array}{l}\text { Time } 2 \text { ( } 3 \text { mc } \\
\text { Measures se } \\
\text { SF-SUNS; D } \\
\text { PES }\end{array}$} \\
\hline & & \\
\hline $\begin{array}{l}\text { Time } 3 \text { (6 months after baseline) } \\
\text { - } \quad \text { Consultation in nurse-led clinic } \\
\text { - } \quad \text { Discuss previously identified needs } \\
\text { - } \quad \text { Apply measures: SF-SUNS; DASS2 } \\
\text { - Encourage self-empowerment strate } \\
\text { - } \quad \text { Encourage follow-up with GP }\end{array}$ & Mac; PES & $\begin{array}{l}\text { Time } 3(6 \mathrm{mc} \\
\text { Measures se } \\
\text { SF-SUNS; D } \\
\text { PES }\end{array}$ \\
\hline $\begin{array}{l}\text { Interview }(\mathrm{N}=10) \\
\text { - Assessment of perception of nurse- } \\
\text { led lymphoma survivorship clinic }\end{array}$ & $\begin{array}{l}\text { GP evalu } \\
\text { - Evalı } \\
\text { use }\end{array}$ & $\begin{array}{l}(\mathrm{N}=30) \\
\text { of SCP TS }\end{array}$ \\
\hline
\end{tabular}

onto a master coding sheet and assigned a numerical identifier code after randomisation.

\section{SCP and TS}

An extensive review of the literature ${ }^{38}$ and available SCPs and TS was undertaken. Many institutions in Australia are using US-based templates that are large (up to 20 pages), not tailored to the individual and provide resources that are not contextualised to the Australian healthcare setting. Therefore, we developed a lymphoma SCP TS in collaboration with a haematology consultant, GP and other multidisciplinary team members (eg, consumers, psychologists, cancer nurses and academic cancer researchers). This has been created as a word document template to be filled in by the nurse. The perspectives of lymphoma survivors $(n=6)$ and clinicians (including GPs; $n=6$ ) were sought to determine the relevance of the proposed SCP TS items. Each item was assessed for content and apparent internal consistency (whether items should be included and the general fit with other items) using either yes or no responses to the items. Content validity used a rating scale ( $1=$ not relevant to $4=$ highly relevant). The content validity index $(\mathrm{CVI})^{47}$ was generated for each item by adding the number of 'yes' scores (content, clarity and apparent internal consistency) and scores of 3 or 4 (content validity). The mean CVI consumer results were as follows: clarity 0.98; apparent internal consistency 100; content validity 0.95. Consumers demonstrated complete agreement of 1.0 for internal consistency items. The mean CVI clinician results were as follows: clarity 0.99; apparent internal consistency 0.95; content validity 0.84 . Feedback in the comments section of the evaluation interestingly indicated GPs did not value or 
require a large TS document. Consensus of the research team was reached for the TS (half a page in length) and SCP (one and a half pages in length).

The TS is completed using existing medical record information such as diagnosis, treatment, complications and use of allied health providers. The first section of the SCP includes a table for the inclusion of individualised potential late effects. This table comprises the late effect; information for the GP about tests or follow-up required and when; and the symptomology the participant needs to be aware of, with encouragement to follow these up with the GP. Prior to recruitment, a comprehensive list of potential late effects and follow-up required was developed for each lymphoma type using available published literature and guidelines (KT). This list was circulated, discussed and amended by the haematologists who were aware these would be used to guide their population of the table. Tailored individualised potential late effects will be documented based on treatments administered, participants' demographics and health characteristics. Once the TS and this aspect of the SCP are completed, it will be emailed to the haematologist for final approval. Once amendments (if any) are made, the haematologist signs the TS. The second page of the SCP is patient centred and populated by the nurse in consultation with the participant. Participants will be asked to identify three main concerns, health goals and proposed actions to achieve these goals.

\section{Sample size}

The calculation of a sample size is not required for pilot RCTs as effect size is not yet known. Rather the purpose of the pilot is to determine variability in measures from which effect sizes can be calculated. Approximately 75 patients are seen per year at the study setting; however, this figure is inclusive of new and existing patients. Therefore, a consecutive sample of 60 participants will be recruited and randomised 1:1 to either control or intervention group (30 participants are expected in each group). It is necessary to establish test-retest reliability for the SF-SUNS by demonstrating a minimum intraclass correlation (ICC) of 0.8. Therefore, a sample size of 39 (rounded up to 40 participants) administered on two consecutive occasions no more than 5 days apart (baseline and 5 days later) is required to achieve $80 \%$ power to detect this ICC of 0.8 . $^{48}$

\section{Patient-reported outcome measures}

A review of the literature ${ }^{44}$ has resulted in four assessment instruments being selected to measure the outcomes proposed: SF-SUNS; Depression Anxiety Stress Scale (DASS21); Mini-Mental Adjustment to Cancer Scale (Mini-MAC); and Patient Empowerment Scale (PES). These instruments have demonstrated reliability and validity with haematological cancer survivors as shown in table 1.

\section{Baseline data collection}

Baseline data collection from consenting participants will occur 3 months after treatment completion. All participants will self-report demographic information and complete the four assessment instruments. In addition, they will receive a second SF-SUNS instrument to complete no later than 5 days after the baseline testing. These will be returned via a reply-paid envelope to allow the researchers to undertake test-retest reliability testing. Medical demographic information obtained will include type of haematological cancer, stage of disease, type of treatment received (chemotherapy, immunotherapy, radiotherapy), date of diagnosis, time since diagnosis, treatment complications or dose modifications, and comorbidities. Personal demographic information collected will include sex, age, marital status, age of children (if any), postcode, occupation, income level, education level and health behaviours such as smoking, alcohol consumption and weight.

\section{Randomisation}

After baseline assessment, participants will be randomised to either the current standard of care or intervention group. Computer-generated random numbers using a four-digit sequence have been generated and linked to group allocation by an independent statistician. An independent member of the research team, to ensure confidentiality and offset bias in randomisation, has sealed a hard copy of each individual number and group in an opaque envelope. The envelopes are consecutively numbered and will be distributed to consenting participants in this order. Control group participants will be made aware that another researcher will follow-up nonquestionnaire return with a telephone call to the participant after 2 weeks.

\section{Control group}

Control group participants will receive follow-up care as per haematologists' usual practice. At 3 and 6 months after baseline, the same four assessment instruments will be sent to the participant, and they will self-report any issues or unmet supportive care needs. An addressed reply paid envelope will be provided to return assessments. Participants who score high unmet needs will be encouraged to discuss these with their haematologist at their usual follow-up appointment.

\section{Intervention group}

Following baseline data collection, intervention group participants will have an appointment at the nurse-led lymphoma survivorship clinic. The first page of the SCP TS will be populated prior to this appointment. At the first nurse-led lymphoma survivorship clinic, any concerns the participant has regarding the end of treatment will be discussed and normalised. The nurse will discuss the TS and potential late effects. The second page of the SCP will be completed by the nurse using an electronic template in collaboration with the participant. At this 
Table 1 Outcomes assessment instruments

\begin{tabular}{|c|c|c|c|c|}
\hline Instrument & Use & Items and factors & Internal consistency & Additional issues \\
\hline $\begin{array}{l}\text { Short-Form Survivor } \\
\text { Unmet Needs Survey } \\
\text { (SF-SUNS) }\end{array}$ & $\begin{array}{l}\text { Developed for cancer survivors to } \\
\text { assess unmet needs. Assess the gap } \\
\text { between patient self-reported } \\
\text { concerns and the level of support they } \\
\text { require } \\
\text { Discriminates between survivors at } \\
\text { different stages post-treatment } \\
\text { completion }\end{array}$ & $\begin{array}{l}30 \text { items }-0 \text { (no unmet need) to } 4 \text { (very } \\
\text { high unmet need } \\
\text { Four factors: information ( } 3 \text { items); } \\
\text { financial concerns ( } 8 \text { items); access and } \\
\text { continuity of care ( } 6 \text { items); relationships } \\
\text { and emotional health ( } 13 \text { items) }\end{array}$ & $\begin{array}{l}\text { Cronbach's } \alpha \text { scores for all } \\
\text { domains were } \geq 0.85 \\
\text { intraclass correlation (ICC) } \\
\text { across all domains high, ie, } \\
\geq 0.9 \text { indicating SF-SUNS } \\
\text { reliably measured the level of } \\
\text { unmet need }\end{array}$ & $\begin{array}{l}\text { Test-retest reliability not } \\
\text { established } \\
\text { Will be undertaken } \\
\text { during this study }\end{array}$ \\
\hline $\begin{array}{l}\text { Depression Anxiety } \\
\text { Stress Scale } \\
\text { (DASS-21 }^{50}\end{array}$ & $\begin{array}{l}\text { Measures multiple dimensions of } \\
\text { depression, anxiety and stress }\end{array}$ & $\begin{array}{l}\text { Three } 7 \text {-item scales-0 (did not apply to } \\
\text { me at all) to } 3 \text { (applied to me very much, } \\
\text { or most of the time) } \\
5 \text { subscale severity ratings: normal, mild, } \\
\text { moderate, severe and extremely severe }\end{array}$ & $\begin{array}{l}\text { Cronbach's } \alpha \text { subscale scores } \\
\text { were: } 0.94 \text { depression; } 0.87 \\
\text { anxiety; and } 0.91 \text { stress }{ }^{51}\end{array}$ & $\begin{array}{l}\text { Used to support SUNS } \\
\text { psychometric properties } \\
\text { in haematology cancer } \\
\text { survivors }\end{array}$ \\
\hline $\begin{array}{l}\text { Mini-Mental } \\
\text { Adjustment to Cancer } \\
\text { Scale (Mini-MAC) }\end{array}$ & $\begin{array}{l}\text { Measures cancer-specific coping } \\
\text { strategies }\end{array}$ & $\begin{array}{l}29 \text { items- } 5 \text { cancer-specific coping } \\
\text { strategies: helplessness-hopelessness } \\
\text { ( } 8 \text { items); anxious preoccupation ( } 8 \text { items); } \\
\text { fighting spirit ( } 4 \text { items); cognitive } \\
\text { avoidance ( } 4 \text { items); and fatalism } \\
\text { ( } 5 \text { items). } \\
\text { Scale- } 1 \text { (definitely does not apply to me) } \\
\text { to } 4 \text { (definitely applies to me) }\end{array}$ & $\begin{array}{l}\text { Reliability using Cronbach's } \alpha \\
\text { coefficients for each subscale } \\
\text { ranged from } 0.62 \text { to } 0.88\end{array}$ & $\begin{array}{l}\text { Used with small sample } \\
\text { of haematology cancer } \\
\text { survivors }\end{array}$ \\
\hline $\begin{array}{l}\text { Patient Empowerment } \\
\text { Scale (PES) }{ }^{53}\end{array}$ & $\begin{array}{l}\text { Measures level of patient's coping } \\
\text { ability and self-efficacy in terms of } \\
\text { managing their illness and making } \\
\text { decisions about support strategies }\end{array}$ & 15-item 4-point Likert-type scale & $\begin{array}{l}\text { A high degree of reliability has } \\
\text { been established using the } \\
\text { Rasch Extended Model with the } \\
\text { Person Separation Index of } \\
0.926\end{array}$ & $\begin{array}{l}\text { Used in haematology/ } \\
\text { oncology patients }\end{array}$ \\
\hline
\end{tabular}


time, the importance of follow-up recommendations will be emphasised. The SCP will then be printed, signed and dated by the participant and the nurse. The completed SCP TS will then be copied, with the original given to the participant, a copy placed in the participant's medical records and a copy sent to their GP. Motivational interviewing techniques will be employed for healthy lifestyle behaviours and to assess for readiness to make behavioural change. Participants will be encouraged to identify and explore behaviours they would like to modify using a chart that enables them to list likes and dislikes of specific behaviours and potential impacts of perceived behavioural change. By listening to concerns, highlighting conflicts arising from behaviour and documenting on the chart will potentially enable participants to assume control of decision-making related to behavioural change. Participants will be encouraged to set realistic time frames and identify habits and beliefs that may possibly be hindering change. Tailored evidenced-based information and advice in a resource pack will then be issued. It is anticipated that a consultation of $60 \mathrm{~min}$ will be required in a private clinic room.

A further two appointments will be made at 3 and 6 months after baseline, where the same four assessment instruments will be completed by the participant, and they will self-report any issues or unmet supportive care needs. These will be discussed and the appropriate resources, support and information provided. Participants will be encouraged to discuss their health concerns, goals and progress with any action they may have taken. Participants will be asked if they have seen their GP in the last 3 months and if they took the SCP TS and discussed any of the late-effect screening recommendations, their participant-identified concerns or goals. This will aid the transition to GP follow-up where the benefits of shared care will be explained. A checklist for each participant of the resources provided will be kept.

\section{DATA ANALYSIS}

Quantitative data will be analysed using univariate and multivariate statistical techniques with SPSS data analysis software. Descriptive statistics will be used to analyse the demographic variables collected. Responses to the SF-SUNS, DASS21, Mini-MAC and PES will be scored according to the algorithms in the instrument manuals. Measures from all instruments will be checked for normal variance within the two groups. Within each group, paired t test comparisons will be made between baseline measurements and at each time point: baseline, 3 months and 6 months. Differences between intervention and control groups will then be assessed at each time point. Test-retest reliability using ICC will be undertaken on the SF-SUNS instrument. The minimum ICC value required for this scale is 0.8 . Participants who drop out or are lost to follow-up or need to be excluded after the start will be accounted for by intention-to-treat analyses. CIs will reflect the contrast between groups to show treatment effect. Missing data, incomplete answers and non-response will be recorded.

\section{Qualitative interviews}

Supplementary in-depth, semistructured interviews will occur with $\sim 10$ consenting participants when they have completed all intervention components (after 6 months). This number will allow for saturation of themes. ${ }^{54-56}$ Telephone interviews will be digitally recorded and undertaken by an independent researcher to ensure participants are given the opportunity to freely express positive and negative perceptions of their experience. The use of a qualitative approach will provide depth of information regarding the personal impact of the nurse-led lymphoma survivorship clinic on the participant. The interviews will also highlight any issues or challenges for this group that could be better addressed in the future.

Interviews will be transcribed verbatim and thematic analysis used to determine themes and patterns within the text. ${ }^{57-59}$ QSR NVivo qualitative analysis data management software will be used to manage interview data.

\section{GP evaluations}

A non-validated evaluation will be sent to GPs who have received the SCP TS. This was developed in consultation with a GP and will ascertain if GPs made use of the SCP TS and to elicit perceptions of the value and effectiveness of this document in facilitating communication between the treating hospital and GP, and GP and participant. This will guide future refinement of the SCP TS. Analysis will use descriptive statistics and distribution analysis techniques. Open-ended questions will use content analysis techniques. GPs will be called by the researcher after 2 weeks for non-return of the questionnaire to remind them to fill in and return the evaluation in the reply-paid envelope.

\section{DISCUSSION}

A significant culture change is required for providers to recognise survivorship care as a standard component of quality cancer care that involves all health professionals, participants and families. The gap in knowledge contributes to a current model of survivorship care that is fragmented, with inadequate service provision at treatment completion, leading to unmet needs along the survivorship continuum. ${ }^{60}$ The cancer specialist is not necessarily required for routine screening and follow-up. However, the involvement of other health professionals, including primary care, necessitates the need for an awareness of the treatment delivered and the long-term and late-effect risks. ${ }^{8}$

This study will address the lack of robust empirical evidence in haematology survivorship care. A nurse-led model of care would assist patients transitioning from the end of treatment to the survivorship phase. Furthermore, the provision of an individualised SCP TS 
is a means to empower individuals with knowledge about their disease and treatment and to assume responsibility for future surveillance and disease management. It will likewise take advantage of 'teachable moments' at the end of active treatment to support and promote patient participation in healthy lifestyle behaviours. ${ }^{38}$ This is particularly vital for younger survivors, given the expectation of a longer survivorship period. ${ }^{40}$

The intervention has been timed to occur in the early survivorship phase. This has been supported by preliminary focus group work including lymphoma cancer survivors who indicated they often felt abandoned at treatment completion. ${ }^{22}$ This timing also concords with McDowell et al, ${ }^{29}$ who found assessments and interventions undertaken in the early survivorship phase (up to 2 years post diagnosis) led to fewer unmet needs moving into the extended survivorship phase (over 5 years).

The CALy trial will examine the impact and effectiveness of the nurse-led lymphoma survivorship clinic intervention through an assessment of the important clinical outcomes: unmet informational and practical needs; depression, anxiety and stress; coping; and selfempowerment as measured by the instruments chosen. It is therefore designed to improve the identification of unmet needs. Testing of such an intervention by an RCT has not been published in lymphoma survivorship studies to date. Consequently, it will make a significant contribution to the planning and delivery of survivorship care. Likewise, it represents a substantial and original contribution to knowledge and support for haematology survivorship care as few studies aim to improve the psychosocial and supportive care of this cohort. If the intervention achieves its intended outcomes, it may potentially lead to the development of nurse-led haematology survivorship clinics across the tertiary health sector in Western Australia that could ultimately be expanded to all cancer survivors.

\section{Ethics}

Ethics approval has been gained from the relevant hospital (2015-020) and university (015007F) Human Research Ethics Committees (HRECs). The trial is registered at the Australian and New Zealand Clinical Trials Registry (ACTRN 1261500530527) and the Western Australia Cancer Clinical Trials Registry. The trial is open to patient recruitment. It is not expected participants will be exposed to any undue risks or harm by participation. Participant information will remain confidential and deidentified where appropriate. Economic harm will be minimised by providing appointments when the participant is already attending the hospital. Exploring concerns may be distressing and if this occurs, participants will be referred to the appropriate counselling services as per usual clinical practice. Collected data will be securely stored at the university for 15 years after study completion and will only be accessible with written permission from the researcher and relevant university and hospital sites.

\section{Dissemination}

We plan to complete the study by December 2017 and report trial results in 2018. It is anticipated the main trial outcomes will be published in a single paper in a refereed cancer journal. Further publications will explore the qualitative data and the test-retest reliability measures of the SF-SUNS. We will correspondingly present findings at national and international conferences.

\section{Author affiliations}

${ }^{1}$ Western Australia Cancer and Palliative Care Network, Perth, Western Australia, Australia

${ }^{2}$ School of Nursing and Midwifery and Centre for Nursing and Midwifery Research, University of Notre Dame Australia, Fremantle, Western Australia, Australia

${ }^{3}$ Sir Charles Gairdner Hospital, Nedlands, Western Australia, Australia ${ }^{4}$ School of Medicine, University of Western Australia, Crawley, Western Australia, Australia

${ }^{5}$ Institute for Health Research, University of Notre Dame Australia, Fremantle, Western Australia, Australia

${ }^{6}$ St John of God Hospital, Murdoch, Western Australia, Australia

${ }^{7}$ School of Nursing, Edith Cowan University, Joondalup, Western Australia, Australia

Contributors KT contributed to the literature reviews and study design, was involved in all aspects of protocol and interventions and the overall preparation and writing of the manuscript. She is undertaking this research as part of her PhD. LM is the principal investigator of the study and the principal PhD supervisor of KT. LM obtained the grant funding to support the study and has led the development and contributed to all aspects of the study, including design; protocol and interventions; manuscript preparation and revision. DJ contributed the original concept for this study and has participated in all aspects of the design, protocol and intervention development, manuscript preparation and revision. MB has contributed to the study's research questions, methodology, data analysis plan, manuscript preparation and revision. CB has contributed to the study's qualitative component, methodology, manuscript preparation and revision. All authors have been involved in drafting and critical evaluation of this manuscript. All authors have read and approved the final version.

Funding This study is supported through the University of Notre Dame Australia (Fremantle) Australian Government's Collaborative Research Networks (CRN) program.

Competing interests None declared.

Ethics approval Sir Charles Gairdner Hospital HREC 2015-020 and University of Notre Dame Australia HREC 015007F.

Provenance and peer review Not commissioned; externally peer reviewed.

Open Access This is an Open Access article distributed in accordance with the Creative Commons Attribution Non Commercial (CC BY-NC 4.0) license, which permits others to distribute, remix, adapt, build upon this work noncommercially, and license their derivative works on different terms, provided the original work is properly cited and the use is non-commercial. See: http:// creativecommons.org/licenses/by-nc/4.0/

\section{REFERENCES}

1. American Cancer Society. Cancer facts \& figures 2014. Atlanta, GA American Cancer Society, 2014.

2. SEER (Surveillance, Epidemiology and End Results). Cancer statistics review, 1975-2011. National Cancer Institute, 2014.

3. Quaresma M, Coleman MP, Rachet B. 40-year trends in an index of survival for all cancers combined and survival adjusted for age and sex for each cancer in England and Wales, 1971-2011: a population-based study. Lancet 2015;385:1206-18.

4. Australian Institute of Health and Welfare. Cancer in Australia: an overview, 2014. Cancer series no. 90. Cat. no. Can 88. Canberra: AlHW, 2014. 
5. Sant M, Minicozzi P, Mounier M, et al. Survival for haematological malignancies in Europe between 1997 and 2008 by region and age: results of EUROCARE-5, a population-based study. Lancet Oncol 2014:15:931-42.

6. Swash B, Hulbert-Williams N, Bramwell R. Unmet psychosocial needs in haematological cancer: a systematic review. Support Care Cancer 2014:22:1131-41.

7. Carey M, Anderson A, Sanson-Fisher R, et al. How well are we meeting haematological cancer survivors' preferences for involvement in treatment decision making? Patient Educ Couns 2012;88:87-92.

8. Taylor K, Chan RJ, Monterosso L. Models of survivorship care provision in adult patients with haematological cancer: an integrative literature review. Support Care Cancer 2015;23:1447-58.

9. Panek-Hudson Y. Survivorship care-time for innovation? Aust $J$ Cancer Nurs 2013;14:7-12.

10. Aleman BM, van den Belt-Dusebout AW, De Bruin ML, et al. Late cardiotoxicity after treatment for Hodgkin's lymphoma. Blood 2007;109:1878-86.

11. $\mathrm{Ng}$ AK, LaCasce A, Travis LB. Long-term complications of lymphoma and its treatment. J Clin Oncol 2011;29:1885-92.

12. Travis LB, Ng AK, Allan JM, et al. Second malignant neoplasms and cardiovascular disease following radiotherapy. J Natl Cancer Inst 2012;104:357-70.

13. van Leeuwen-Segarceanu EM, Bos WJ, Dorresteijn LD, et al. Screening Hodgkin's lymphoma survivors for radiotherapy induced cardiovascular disease. Cancer Treat Rev 2011;37:391-403.

14. Grinyer A. The late effects of mantle field radiotherapy: the information and support needs of women survivors of Hodgkin's disease. Eur J Oncol Nurs 2010;14:183-9.

15. Hemminki K, Lenner $\mathrm{P}$, Sundquist $\mathrm{J}$, et al. Risk of subsequent solid tumors after non-Hodgkin's lymphoma: effect of diagnostic age and time since diagnosis. J Clin Oncol 2008;26:1850-7.

16. Bishop MM, Lee SJ, Beaumont JL, et al. The preventive health behaviors of long-term survivors of cancer and hematopoietic stem cell transplantation compared with matched controls. Biol Blood Marrow Transplant 2010;16:207-14.

17. Choi M, Sun CL, Kurian S, et al. Incidence and predictors of delayed chronic kidney disease in long-term survivors of hematopoietic cell transplantation. Cancer 2008;113:1580-7.

18. Dicicco-Bloom B, Cunningham RS. The experience of information sharing among primary care clinicians with cancer survivors and their oncologists. J Cancer Surviv 2013;7:124-30.

19. McCabe MS, Jacobs LA. Clinical update: survivorship care-models and programs. Semin Oncol Nurs 2012;28:e1-8.

20. Phillips JL, Currow DC. Cancer as a chronic disease. Collegian 2010;17:47-50.

21. Lobb EA, Joske $D$, Butow $P$, et al. When the safety net of treatment has been removed: patients' unmet needs at the completion of treatment for haematological malignancies. Patient Educ Couns 2009;77:103-8.

22. Monterosso L, Taylor K, Joske D, et al. Defining concerns, unmet needs and support preferences for haematology survivors: an exploratory study to inform intervention development. Fremantle, Western Australia: University of Notre Dame Australia, 2015.

23. Gates $\mathrm{P}$, Seymour JF, Krishnasamy M. Insights into the development of a nurse-led survivorship care intervention for long-term survivors of Hodgkin's lymphoma. Aust J Cancer Nurs 2012;13:4-10.

24. Howell D, Hack TF, Oliver TK, et al. Models of care for post-treatment follow-up of adult cancer survivors: a systematic review and quality appraisal of the evidence. $J$ Cancer Surviv 2012;6:359-71.

25. John C, Armes J. Developing a nurse-led survivorship service for patients with lymphoma. Eur J Oncol Nurs 2013;17:521-7.

26. Cooper JM, Loeb SJ, Smith CA. The primary care nurse practitioner and cancer survivorship care. J Am Acad Nurse Pract 2010;22:394-402.

27. Fitch M. Supportive care framework. Can Oncol Nurs $J$ 2008;18:6-14

28. Ganz PA, Casillas J, Hahn EE. Ensuring quality care for cancer survivors: implementing the survivorship care plan. Semin Oncol Nurs 2008;24:208-17.

29. McDowell ME, Occhipinti S, Ferguson M, et al. Predictors of change in unmet supportive care needs in cancer. Psychooncology 2010;19:508-16.

30. Stricker CT, Jacobs LA, Risendal B, et al. Survivorship care planning after the Institute of Medicine recommendations: how are we faring? $J$ Cancer Surviv 2011;5:358-70.

31. Bodenheimer $\mathrm{T}$, Lorig $\mathrm{K}$, Holman $\mathrm{H}$, et al. Patient self-management of chronic disease in primary care. JAMA 2002;288:2469-75.
32. Kuijpers W, Groen WG, Aaronson NK. A systematic review of web-based interventions for patient empowerment and physical activity in chronic diseases: relevance for cancer survivors. $J$ Med Internet Res 2013;15:e37.

33. Bandura A. Self-efficacy: toward a unifying theory of behavioral change. Psychol Rev 1977;84:191-215.

34. Palmer SC, Jacobs LA, Demichele A, et al. Metrics to evaluate treatment summaries and survivorship care plans: a scorecard. Support Care Cancer 2014;22:1475-83.

35. McCabe MS, Bhatia S, Oeffinger KC, et al. American Society of Clinical Oncology statement: achieving high-quality cancer survivorship care. J Clin Oncol 2013;31:631-40.

36. MacMillan Cancer Support and NHS Improvement. The National cancer survivorship initiative vision. London, UK: Department of Health, 2010.

37. COSA. 2014. http://www.cosa.org.au/media/224898/cancersurvivorship-critical-components-model-for-wellness-draft-20150227. pdf

38. Taylor K, Monterosso L. Survivorship care plans and treatment summaries in adult patients with hematologic cancer: an integrative literature review. Oncol Nurs Forum 2015;42:283-91.

39. Hausman J, Ganz PA, Sellers TP, et al. Journey forward: the new face of cancer survivorship care. J Oncol Pract 2011;7(Suppl 3): e50s-6s.

40. Jabson JM, Bowen DJ. Cancer treatment summaries and follow-up care instructions: which cancer survivors receive them? Cancer Causes Control 2013;24:861-71.

41. Jackson JM, Scheid K, Rolnick SJ. Development of the cancer survivorship care plan: what's next? Life after cancer treatment. Clin J Oncol Nurs 2013;17:280-4.

42. Campbell NC, Murray E, Darbyshire J, et al. Designing and evaluating complex interventions to improve health care. BMJ 2007;334:455-9.

43. Medical Research Council. A framework for development and evaluation of RCTs for complex interventions to improve health 2000

44. Taylor K, Monterosso L. Systematic review of the tools used to assess the informational and practical needs of acute leukaemia and lymphoma survivors. Aust J Cancer Nurs 2016;17:6-13.

45. Moher D, Hopewell S, Schulz KF, et al. CONSORT 2010

Explanation and Elaboration: updated guidelines for reporting parallel group randomised trial. BMJ 2010;340:c869.

46. Schulz KF, Altman DG, Moher D. CONSORT 2010 Statement: updated guidelines for reporting parallel group randomised trials. BMJ 2010;340:c332.

47. Polit $\mathrm{D}$, Beck $\mathrm{C}$. The content validity index: are you sure you know what's being reported? Critique and recommendations. Res Nurs Health 2006;29:489-97.

48. Walter SD, Eliasziw M, Donner A. Sample size and optimal designs for reliability studies. Stat Med 1998;17:101-10.

49. Campbell HS, Hall AE, Sanson-Fisher RW, et al. Development and validation of the Short-Form Survivor Unmet Needs Survey (SF-SUNS). Support Care Cancer 2014;22:1071-9.

50. Lovibond SH, Lovibond PF. Manual for the depression anxiety stress scales, 2nd edn. Sydney: Psychology Foundation University of New South Wales, 1995.

51. Antony MM, Bieling PJ, Cox BJ, et al. Psychometric properties of the 42-item and 21-item versions of the depression anxiety stress scales in clinical groups and a community sample. Psychol Assess 1998;10:176-81.

52. Boyes AW, Girgis A, D'Este C, et al. Flourishing or floundering? Prevalence and correlates of anxiety and depression among a population-based sample of adult cancer survivors 6 months after diagnosis. J Affect Disord 2011;135:184-92.

53. Bulsara C, Styles I. Development of a cancer related patient empowerment scale using the Polytomous Rasch measurment model. Cancer Clin Oncol 2013;2:1-16.

54. Crouch M, McKenzie $\mathrm{H}$. The logic of small samples in interview-based qualitative research. Soc Sci Inf 2006;45:483-99.

55. Onwuegbuzie A, Leech N. A call for qualitative power analyses. Qual Quant 2007;41:105-21.

56. Sandelowski M. Sample size in qualitative research. Res Nurs Health 1995;18:179-83.

57. Grbich C. Qualitative research in health: an introduction. CA: Sage Publications, 1998.

58. Patton MQ. Qualitative research and evaluation methods: integrating theory and practice. SAGE Publications, 2014.

59. Smith JA. Qualitative psychology: a practical guide to research methods. SAGE Publications, 2007.

60. de Leeuw J, Larsson M. Nurse-led follow-up care for cancer patients: what is known and what is needed. Support Care Cancer 2013;21:2643-9. 